\title{
Compliance and barriers to growth hormone therapy in children
}

\author{
Liudmyla Senkivska, Mykola Aryayev \\ Odessa National Medical University, Odessa, Ukraine
}

\section{ABSTRACT}

\begin{abstract}
Aim: To compare the level of compliance to recombinant human growth hormone (rGHh) therapy depending on the methods of its measuring and identify social/demographic, cognitive/emotional and medical factors (barriers) that influence adherence to treatment of children with growth hormone deficiency (GHD).

Material and methods: The study group included 80 children with GHD at the age of $7.1 \pm 0.4$ years. The assessment of adherence to rGHh therapy was carried out by the self-report method and the Morisky Medication Adherence Scale (MMAS). Social/demographic, cognitive/emotional, and medical factors were considered as potential barriers compliance. The categorical variables were expressed as frequency (percentage) and analyzed by $\chi^{2}$-test. A $p$-value $<0.05$ was considered as statistically significant.

Results: Comparison of two methods of measuring adherence to rGHh therapy demonstrates the advantages of using the MMAS. The self-report method overestimates the compliance . Children with unacceptable adherence were more likely to have such social/demographic factors as below average income, rGHh shortage and being away from home. Cognitive/emotional barriers to compliance included forgetfulness/ preoccupation, fear of injections, and treatment fatigue. Medical factors that impeded the development of acceptable compliance included insufficient patient/parents counseling, use of conventional syringe instead of automatic pen devices. Implementation of the compliance support program increased the frequency of acceptable (high and medium) adherence to rGHh therapy to $80.0 \%$ compared to the baseline level of $57.4 \%(p=0.002)$.

Conclusions: Adequate assessment of compliance to rGHh therapy depends on the choice of measurement method. An individual compliance support program based on the results of identification of barriers contributes to an increase in the adherence to rGHh therapy in children with GHD.
\end{abstract}

KEY WORDS:

compliance, children, growth hormone therapy, barrier factors.

\section{INTRODUCTION}

Patient compliance (adherence) to therapy has medical and bioethical dimension. From the standpoint of medicine, acceptable compliance to therapy improves its results, especially in chronic diseases [1]. From the point of view of bioethics, compliance to therapy testifies to a good partnership between the doctor, child, parents and demonstrates respect for patient autonomy [2]. It is known that low compliance to recombinant human growth hormone (rGHh) therapy in children with growth hormone deficiency (GHD) reduces the growth rate and is one of the reasons for the suboptimal growth response [3]. Prevalence of non-adherence to rGHh therapy in paediatric patients varied from 5 to $82 \%$, depending on the methods and definitions used [4]. Formation of compliance to rGHh therapy in children with GHD is a multifactorial process with regional characteristics. [5]. Until now, studies of compliance to rGHh therapy in children with GHD have not been conducted in Ukraine, and

\section{ADDRESS FOR CORRESPONDENCE:}

Mykola Aryayev, Odessa National Medical University, Odessa, Ukraine, e-mail: aryayev.nl@ukr.net 
regional barriers to its formation have not been identified. Considering the aforementioned, the study objectives were to compare the level of compliance to $\mathrm{rGHh}$ therapy using different methods of its measuring and to identify social/demographic, cognitive/emotional and medical barriers that influence adherence to treatment in children with GHD.

\section{MATERIAL AND METHODS}

The study was approved by the Odessa Regional Children's Clinical Hospital Ethical Committy and performed in accordance with the standards of good clinical practice and the principles of Declaration of Helsinki (Revised 2013). Written informed consent from parents was obtained. The study group included 80 children with GHD at the age of $7.1 \pm 0.4$ years (height SDS $-3.3 \pm 0.1$; bone age $4.4 \pm 0.3$ years). Adherence to rGHh therapy was assessed using the self-report method (drug administration diary) and the Morisky Medication Adherence Scale (MMAS). The baseline adherence to therapy was established over the last month of follow-up. The diary was completed on a daily basis and checked by a physician during quarterly visits. According to the results of self-report, compliance was considered high when the patient declared 90-100\%, medium when receiving $85-90 \%$ and low when receiving $80-85 \%$ of the prescribed injections during the last month. The assessment of compliance to rGHh therapy according to the MMAS scale was based on data from 8 items of the questionnaire. Adherence was considered high if the child received 8 points, medium when receiving 6.0-7.9 points and low in the case of $<6$ points. Children with acceptable adherence (i.e. high and medium) were considered compliant to rGHh therapy. Children with unacceptable adherence (i.e. low or no adherence) were considered non-compliant to rGHh therapy [6]. The baseline data were compared with those obtained after the adherence support program. The time between implementing the individual support compliance program and retesting was 6 months. The statistical difference between the two proportions, $95 \%$ confidence interval (CI) for this difference, $\chi$ and Student criterion were calculated using the Internet calculator SISA (Simple Interactive Statistical Analysis; http://quantativeskills.com/). The $p$-value $\leq 0.05$ was considered to be statistically significant.

\section{RESULTS}

The research hypothesis was the assumption about the dependence of compliance level on the methods of measuring and the importance of a number of medical, cognitive/emotional and social/demographic factors as barriers to adherence in GHD children to rGHh therapy. The prerequisite for comparing compliance levels depending on the method of its measurement was data on significant fluctuations in the compliance rate in children with GHD [7]. Compliance level determined by the self-report method and the MMAS scale revealed significant differences in the results (Table 1). According to self-report data, acceptable adherence (high and medium) was noted in $95.2 \%$ (95\% CI: $90.5 \div 99.9)$ of children with GHD. At the same time, the MMAS score established

TABLE 1. Frequency of acceptable and unacceptable adherence to rGHh therapy according to the results of the self-report method and the MMAS

\begin{tabular}{|l|c|c|c|c|}
\hline \multirow{2}{*}{$\begin{array}{l}\text { Methods of measuring } \\
\text { adherence to GH therapy }\end{array}$} & \multicolumn{2}{|c|}{ Acceptable compliance } & \multicolumn{2}{|c|}{ Unacceptable compliance } \\
\cline { 2 - 5 } & High & Medium & Low & Absent \\
\cline { 2 - 5 } & $n ; \%(95 \% \mathrm{Cl})$ & $n ; \%(95 \% \mathrm{Cl})$ & $n ; \%(95 \% \mathrm{CI})$ & $n ;(95 \% \mathrm{Cl})$ \\
\hline Self-report & $28 ; 35.2 \%(24.7 \div 45.7)$ & $48 ; 60.0 \%(49.3 \div 70.7)$ & $4 ; 4.8 \%(0.1 \div 9.5)$ & $0 ; 0.0 \%$ \\
\hline MMAS & $13 ; 16.6 \%(8.4 \div 24.8)$ & $33 ; 40.8 \%(23.1 \div 43.7)$ & $34 ; 42.6 \%(31.8 \div 53.4)$ & $0 ; 0.0 \%$ \\
\hline
\end{tabular}

${ }^{*} n$ - absolute number of children; \% - relative number of children; $95 \%$ Cl - 95\% confidential interval

TABLE 2. Potential social/demographic barriers to growth hormone therapy adherence

\begin{tabular}{|c|c|c|c|c|}
\hline$N=80$ & $\begin{array}{c}\text { Acceptable compliance (46) } \\
\qquad ; \% \%(95 \% \mathrm{Cl})\end{array}$ & $\begin{array}{l}\text { Unacceptable compliance (34) } \\
\qquad n ; \%(95 \% \mathrm{Cl})\end{array}$ & $\chi^{2}$ & $P$ \\
\hline Age $<7$ years & $23 ; 50.0(35.6 \div 64.4)$ & $18 ; 52.9(36.1 \div 69.7)$ & 0.07 & 0.80 \\
\hline Gender (male) & $29 ; 63.0(49.0 \div 77.0)$ & $27 ; 79.4(19.2 \div 51.4)$ & 2.47 & 0.12 \\
\hline Below average income & $3 ; 6.5(-0.6 \div 13.6)$ & $8 ; 23.5(9.2 \div 37.8)$ & 4.71 & 0.03 \\
\hline $\begin{array}{l}\text { Parents' education below } \\
\text { secondary }\end{array}$ & $12 ; 26.1(13.4 \div 38.8)$ & $14 ; 41.2(24.7 \div 57.7)$ & 2.01 & 0.16 \\
\hline RGHh shortage & $1 ; 2.2(-2.0 \div 6.4)$ & $5 ; 14.7(2.8 \div 26.6)$ & 4.34 & 0.04 \\
\hline Being away from home & $3 ; 6.5(-0.6 \div 13.6)$ & $8 ; 23.5(9.2 \div 37.8)$ & 4.71 & 0.03 \\
\hline
\end{tabular}

$*_{n}$ - absolute number of children; \% - relative number of children; $95 \% \mathrm{Cl}-95 \%$ confidential interval; $p$-value $\leq 0.05$ is considered to be statistically significant 
TABLE 3. Potential cognitive/emotional barriers to growth hormone therapy adherence

\begin{tabular}{|l|c|c|c|c|}
\hline$N=80$ & $\begin{array}{c}\text { Acceptable compliance (46) } \\
n ; \%(95 \% \mathrm{Cl})\end{array}$ & $\begin{array}{c}\text { Unacceptable compliance (34) } \\
n ; \%(95 \% \mathrm{CI})\end{array}$ & $\chi^{2}$ & $P$ \\
\hline Forgetfulness/preoccupation & $1 ; 2.2(-2.0 \div 6.4)$ & $5 ; 14.7(2.8 \div 26.6)$ & 4.34 & 0.04 \\
\hline Painful injection & $2 ; 4.3(-1.6 \div 10.2)$ & $7 ; 20.6(7.0 \div 34.2)$ & 5.15 & 0.02 \\
\hline Dissatisfaction with treatment results & $1021.7(9.8 \div 33.6)$ & $13 ; 38.2(21.9 \div 54.5)$ & 2.57 & 0.11 \\
\hline Negative attitude of friends or family & $1 ; 2.2(-2.0 \div 6.4)$ & $2 ; 5.9(-2.0 \div 13.8)$ & 0.73 & 0.39 \\
\hline Treatment fatigue & $2 ; 4.3(-1.6 \div 10.2)$ & $6 ; 17.8(4.9 \div 30.7)$ & 3.90 & 0.05 \\
\hline
\end{tabular}

${ }_{n} n$ - absolute number of children; \% - relative number of children; $95 \%$ Cl - 95\% confidential interval; $p$-value $\leq 0.05$ is considered to be statistically significant

TABLE 4. Potential medical barriers to growth hormone therapy adherence

\begin{tabular}{|l|c|c|c|c|}
\hline$N=80$ & $\begin{array}{c}\text { Acceptable compliance (46) } \\
n ; \%(95 \%(\mathrm{Cl})\end{array}$ & $\begin{array}{c}\text { Unacceptable compliance (34) } \\
n ; \%(95 \% \mathrm{CI})\end{array}$ & $\chi^{2}$ & $P$ \\
\hline Inadequate contact with health care providers & $3 ; 6.5(-0.6 \div 13.6)$ & $4 ; 11.8(1.0 \div 22.6)$ & 0.68 & 0.41 \\
\hline Insufficient patient/parents counseling & $2 ; 4.3(-1.6 \div 10.2)$ & $9 ; 26.5(11.7 \div 41.3)$ & 8.03 & 0.005 \\
\hline Concerns about long-term therapy & $4 ; 8.7(0.6 \div 16.8)$ & $5 ; 14.7(2.8 \div 26.6)$ & 0.70 & 0.40 \\
\hline Adverse effects & $2 ; 4.3(-1.6 \div 10.2)$ & $3 ; 8.8(-0.7 \div 18.3)$ & 0.67 & 0.41 \\
\hline Comorbid diseases & $1 ; 2.2(-2.0 \div 6.4)$ & $1 ; 2.9(-2.7 \div 8.5)$ & 0.04 & 0.84 \\
\hline Injection not given by the child & $3 ; 6.5(-0.6 \div 13.6)$ & $2 ; 5.9(-2.0 \div 13.8)$ & 0.01 & 0.91 \\
\hline Use of syringe instead of automatic pen devices & $30 ; 65.2(51.4 \div 79.0)$ & $20 ; 43.5(26.8 \div 60.2)$ & 3.69 & 0.05 \\
\hline
\end{tabular}

${ }^{*} n$ - absolute number of children; \% - relative number of children; $95 \% \mathrm{Cl}-95 \%$ confidential interval; $p$-value $\leq 0.05$ is considered to be statistically significant

TABLE 5. The impact of the adherence support program on the level of compliance to rGHh therapy

\begin{tabular}{|l|c|c|c|c|}
\hline Compliance & $\begin{array}{c}\text { Baseline } \\
n ; \%(95 \% \mathrm{Cl})\end{array}$ & $\begin{array}{c}\text { Program results } \\
n ; \%(95 \% \mathrm{Cl})\end{array}$ & $\chi^{2}$ & $P$ \\
\hline High & $13 ; 16.2(8.1 \div 24.3)$ & $24 ; 30.0(20.0 \div 40.0)$ & 4.26 & 0.04 \\
\hline Medium & $33 ; 41.2(30.4 \div 52.0)$ & $40 ; 50.0(39.0 \div 61.0)$ & 1.24 & 0.26 \\
\hline Low & $34 ; 42.6(23.6 \div 44.4)$ & $16 ; 20.0(11.2 \div 28.8)$ & 9.44 & 0.002 \\
\hline
\end{tabular}

${ }^{*} n$ - absolute number of children; \% - relative number of children; $95 \% \mathrm{Cl}-95 \%$ confidential interval; $p$-value $\leq 0.05$ is considered to be statistically significant

acceptable adherence only in 57.4\%; (95\% CI: 46.6 $\div 68.2)$ of patients with GHD. Taking into account the overestimation of the frequency of acceptable compliance and underestimation of the frequency of unacceptable compliance when using the self-report method, the study of adherence barriers to rGHh therapy was carried out solely on the basis of the MMAS scale. Unacceptable adherence to therapy was the reason for identifying and eliminating removable barriers to the compliance and/or increasing the dose of rGHh.

The relationship between the level of compliance and the action of a number of social/demographic, cognitive/emotional and medical factors, which could be barriers to the implementation of the prescribed therapy, has been studied. Among the social/demographic factors, below average income, rGHh shortage, frequent child's being away from home were more often detected in the group of children with unacceptable adherence to rGHh therapy (Table 2). The formation of unacceptable adherence was facilitated by such emotional and cognitive factors as forgetting of prescriptions, fear of injections, and fatigue from treatment (Table 3). Among the medical factors associated with unacceptable adherence, insufficient patient/parents counseling and use of conventional syringe instead of automatic pen devices were identified as compliance barriers (Table 4 ).

The identified barriers to adherence were taken into account when compiling the individual program to support compliance to rGHh therapy in children with GHD. In cases of low income or limited access to the drug, possible social assistance and inclusion in the state program of free provision of growth hormone were provided. The risks of interruption of $\mathrm{rGHh}$ therapy due to the child being away of home were discussed, In cases of child's forgetfulness/preoccupation, methods of reminding about the administration of the drug were suggested. The benefits of high adherence to $\mathrm{rGHh}$ therapy were discussed with children and parents in cases of fear of injections and treatment fatigue. Attention was paid to the development of patient/ parents counseling and to the introduction of contemporary methods of the drug administration. After the implementation of the adherence support program, an increase in the frequency of acceptable (high and medium) compliance to rGHh therapy was noted: $80.0 \%(95 \% \mathrm{CI}: 71.2 \div 88.8)$ compared with

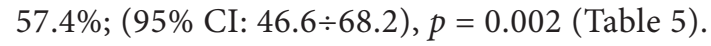




\section{DISCUSSION}

The obtained results clarify the interpretation of significant fluctuations in the rank of compliance to $\mathrm{rGHh}$ therapy in children with GHD, which apparently were related to the differences in the definition and measurement of adherence. Similar fluctuations in compliance have also been found in patients with diabetes and hypertension [8]. Although the self-report method is not costly, it poorly detects unacceptable adherence to rGHh therapy and overestimates the level of compliance. It would probably be more informative to analyze the number of missed injections per week in the context of a separate study. Overestimation of adherence according to self-report data may be associated with bias of children and parents and the desire to avoid confrontation with medical personnel due to non-fulfillment of appointments. When using the MMAS method, the effect of such factors is less pronounced due to the indirect nature of the questions. The results of the study show the inappropriateness of using the self-report method to measure the level of compliance to rGHh therapy and make it possible to recommend the MMAS for routine use in clinical practice in Ukraine. A significant level of non-compliance with rGHh therapy in the examined children with GHD is consistent with the results of a number of studies and determines the need to improve methods for measuring compliance and implementing individual compliance support programs [9]. The developed compliance support program included wide range of potentially modifiable social/demographic, cognitive/emotional and medical factors that influence adherence to $\mathrm{rGHh}$ therapy. Some factors identified in other studies were also taken into account [10]. It was surprising that the satisfaction or dissatisfaction of the treatment results was not an important factor for the compliance. This finding can be presumably associated with a relatively short observation period. Implementation of the adherence support programs reduces the incidence of non-compliance of children with GHD to rGHh therapy from $42.6 \%$ to $20.0 \%$ according to the MMAS. The re-evaluation of the self-report diary was not recommended. Prospects for further research include the development of adherence-focused interventions with the aim of improving the use of prescribed treatment in children with GHD.

\section{CONCLUSIONS}

1. The level of compliance to rGHh therapy in children with GHD depends on the applied methods of measuring. The choice of an adequate measurment methodology is an important task in the context of identifying adherence barriers and developing an individual strategy to support the child and family.

2. Compliance to $\mathrm{rGHh}$ therapy in a child with GHD decreases under the influence of a number of medical, cognitive/emotional, social/demographic factors and their combinations. The combined nature of the adherence barriers to rGHh therapy determines the need for individual complex measures to counteract factors that reduce compliance to treatment in children with GHD.

\section{DISCLOSURE}

The authors declare no conflict of interest.

\section{REFERENCES}

1. Su PH, Yang C, Chao MC, Chiang CL. Monitoring Adherence Rate to Growth Hormone Therapy and Growth Outcomes in Taiwanese Children Using Easypod Connect: Observational Study. JMIR Pediatr Parent 2021; 4: e14774.

2. Neuhaus CP. Global bioethics. Hastings Cent Rep 2017; 47 (6): inside front cover.

3. Centonze C, Guzzetti C, Orlando G, Loche S; Italian ECOS Investigators. Adherence to growth hormone $(\mathrm{GH})$ therapy in naive to treatment GH-deficient children: data of the Italian Cohort from the Easypod Connect Observational Study (ECOS). J Endocrinol Invest 2019; 42: 1241-1244.

4. Fisher BG, Acerini CL. Understanding the growth hormone therapy adherence paradigm: a systematic review. Horm Res Paediatr 2013; 79: 189-196.

5. Mohseni S, Heydari Z, Qorbani M, Radfar M. Adherence to growth hormone therapy in children and its potential barriers. J Pediatr Endocrinol Metab 2018; 31: 13-20.

6. Morisky DE, Ang A, Krousel-Wood M, Ward HJ. Predictive validity of a medication adherence measure in an outpatient setting. J Clin Hypertens (Greenwich) 2008; 10: 348-354.

7. Rosenfeld RG, Bakker B. Compliance and persistence in pediatric and adult patients receiving growth hormone therapy. Endocr Pract 2008; 14: 143-154.

8. Farsaei S, Radfar M, Heydari Z, et al. Insulin adherence in patients with diabetes: risk factors for injection omission. Prim Care Diabetes 2014; 8: 338-345.

9. Graham S, Auyeung V, Weinman J. Exploring Potentially Modifiable Factors That Influence Treatment Non-Adherence Amongst Pediatric Growth Hormone Deficiency: A Qualitative Study. Patient Prefer Adherence 2020; 14: 1889-1899.

10. Graham S, Weinman J, Auyeung V. Identifying Potentially Modifiable Factors Associated with Treatment Non-Adherence in Paediatric Growth Hormone Deficiency: A Systematic Review. Horm Res Paediatr 2018; 90: 221-227. 